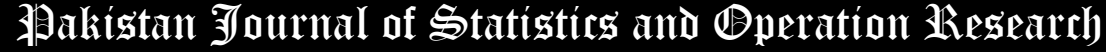

\section{A Study on Moments of Dual Generalized Order Statistics from Exponentiated Generalized Class of Distributions}

\author{
Haseeb Athar ${ }^{1 *}$, Yousef F. Alharbi ${ }^{2}$, Mohamad A. Fawzy ${ }^{2,3}$ \\ * Corresponding Author \\ 1. Department of Statistics and Operations Research, Aligarh Muslim University, Aligarh, India, \\ haseebathar68@gmail.com \\ 2. Department of Mathematics, College of Science, Taibah University, Al Madinah, Kingdom of Saudi Arabia, \\ ymatrafe@taibahu.edu.sa \\ 3. Mathematics Department, Faculty of Science, Suez University, Suez, Egypt, mohfawzy180@yahoo.com
}

\begin{abstract}
In this paper, relations between moments of dual generalized order statistics from a exponentiated generalized class of distributions, given by Cardeiro et al.(2013) are studied. Some particular cases of dual generalized order statistics and examples based on it are discussed. The characterization of given distribution based on moment properties is also presented.
\end{abstract}

Key Words: Dual Generalized Order Statistics; Order Statistics; Record Values; Exponentiated Generalized Distributions; Recurrence Relations; Characterization.

Mathematical Subject Classification: 62G30, 62E10.

\section{Introduction}

The theory of ordered random variables received a tremendous attention from many researchers during the past few decades. This theory deals with the properties and applications of ordered random variables and functions involving them. There are several models of ordered random variables such as order statistics, record values, sequential order statistics, progressively Type-II censored order statistics with interesting applications in many fields of statistical science.

The concept of generalized order statistics is given by Kamps (1995), which unifies the above given models of ordered random variables arranged in ascending order as special case with various choices of the parameters involved. In real life problems sometimes, it happens that the sample is arranged in descending order for example the life length of an electric bulb arranged from highest to lowest. In such situations the distributional properties of variables cannot be studied by using the models of ordered random variables, given by Kamps (1995). The study of distributional properties of such random variables is studied by using the inverse image of generalized order statistics and is popularly known as dual (lower) generalized order statistics (dgos). This concept was first introduced by Pawlas and Szynal (2001) with name lower generalized order statistics and later extensively studied by Burkschat et at. (2003) with name dual generalized order statistics (dgos). The special cases of $d g o s$ are reverse order statistics and lower record values.

During the past centuries, order statistics and related general models of ordered random variables have played immense role in problem of deriving the recurrence relations and identities for single and product moments for some specific as well as general classes of distributions. These moments are widely used in statistical inference. Further, 
these models have also been utilized in the characterization problems. Recurrence relations and identities are important in their own right because they express the higher order moments in terms of the lower order moments and hence make the evaluation of higher order moments easy as well as reduce the time and labour. For more details about theory and applications of ordered random variables, one may refer the book by Shahbaz et al. (2016).

The properties of dgos with reference to recurrence relations between moments and characterization for some specific as well as general class of distributions are studied by several authors in literature, see for example Pawlas and Szynal (2001), Ahsanullah (2004, 2005), Mbah and Ahsanullah (2007), Athar et al. (2010), Khan et al. (2010), Khwaja et al. (2012), Khan and Kumar (2010, 2011), Kumar (2013), Khan and Khan (2015), Noor el al. (2015), Daghistani et al. (2019), Khan et al. (2019), Singh et al. (2021), and references cited therein.

The aim of this paper to present a unified approach to study the moment properties of exponentiated distributions and their generalized forms by considering exponentiated generalized class of distributions, given by Cardeiro et al. (2013).

Definition 1.1: Let $n \geq 2$ be a given integer and $\tilde{m}=\left(m_{1}, m_{2}, \ldots, m_{n-1}\right) \in \square^{n-1}, k>0$ be the parameters such that

$$
\gamma_{i}=k+n-i+\sum_{j=i}^{n-1} m_{j}>0 \text { for } 1 \leq i \leq n-1
$$

The random variables $X_{d}(1, n, \tilde{m}, k), X_{d}(2, n, \tilde{m}, k), \ldots, X_{d}(n, n, \tilde{m}, k)$ are said to be dgos from an absolutely continuous population having cumulative distribution function $(c d f) \quad F()$ and the probability density function $(p d f) f()$, if their joint density function is of the form

$$
\begin{aligned}
f_{X_{d}(1, n, \tilde{m}, k), \ldots, X_{d}(n, n, \tilde{m}, k)}\left(x_{1}, x_{2}, \ldots, x_{n}\right) & \\
= & k\left(\prod_{j=1}^{n-1} \gamma_{j}\right)\left(\prod_{i=1}^{n-1}\left[F\left(x_{i}\right)\right]^{m_{i}} f\left(x_{i}\right)\right)\left[F\left(x_{n}\right)\right]^{k-1} f\left(x_{n}\right)
\end{aligned}
$$

for $F^{-1}(1)>x_{1} \geq x_{2} \geq \ldots \geq x_{n}>F^{-1}(0)$.

Two cases of $d g o s$ are given as:

Case I: $\gamma_{i} \neq \gamma_{j} ; i \neq j=1,2, \ldots, n-1$.

In view of (1) the $p d f$ of $r^{\text {th }} d g o s X_{d}(r, n, \tilde{m}, k)$ is given as

$$
f_{X_{d}(r, n, \tilde{m}, k)}(x)=C_{r-1} f(x) \sum_{i=1}^{r} a_{i}(r)[F(x)]^{\gamma_{i}-1}
$$

and the joint $p d f$ of $r^{\text {th }}$ and $s^{\text {th }} d g o s, X_{d}(r, n, \tilde{m}, k)$ and $X_{d}(s, n, \tilde{m}, k), 1 \leq r<s \leq n$ is

$$
\begin{aligned}
f_{X_{d}(r, n, \tilde{m}, k), X_{d}(s, n, \tilde{m}, k)}(x, y)=C_{s-1}( & \left.\sum_{j=r+1}^{s} a_{j}^{(r)}(s)\left[\frac{F(y)}{F(x)}\right]^{\gamma_{j}}\right) \\
& \times\left(\sum_{i=1}^{r} a_{i}(r)[F(x)]^{\gamma_{i}}\right) \frac{f(x)}{F(x)} \frac{f(y)}{F(y)}, \alpha \leq y<x \leq \beta .
\end{aligned}
$$

where,

$$
C_{r-1}=\prod_{i=1}^{r} \gamma_{i}, \gamma_{i}=k+n-i+\sum_{j=i}^{n-1} m_{j}>0
$$




$$
a_{i}(r)=\prod_{\substack{j=1 \\ j \neq i}}^{r} \frac{1}{\gamma_{j}-\gamma_{i}}, \gamma_{i} \neq \gamma_{j}, 1 \leq i \leq r \leq n
$$

and

$$
a_{i}^{(r)}(s)=\prod_{\substack{j=r+1 \\ j \neq i}}^{s} \frac{1}{\left(\gamma_{j}-\gamma_{i}\right)}, \gamma_{i} \neq \gamma_{j}, r+1 \leq i \leq s \leq n .
$$

Case II: $m_{i}=m($ say $) ; i=1,2, \ldots, n-1$.

The $p d f$ of $r^{\text {th }}$ dgos $X_{d}(r, n, m, k)$ is

$$
f_{X_{d}(r, n, m, k)}(x)=\frac{C_{r-1}}{(r-1) !}[F(x)]^{\gamma_{r}-1} f(x) g_{m}^{r-1}(F(x))
$$

and the joint $p d f$ of $r^{\text {th }}$ and $s^{\text {th }} d g o s, X_{d}(r, n, m, k)$ and $X_{d}(s, n, m, k), 1 \leq r<s \leq n$ is

$$
\begin{aligned}
f_{X_{d}(r, n, m, k), X_{d}(s, n, m, k)}(x, y)=\frac{C_{s-1}}{(r-1) !(s-r-1) !}[F(x)]^{m} f(x) g_{m}^{r-1}(F(x)) \\
\times\left[h_{m}(F(y))-h_{m}(F(x))\right]^{s-r-1}[F(y)]^{\gamma_{s}-1} f(y), \alpha \leq y<x \leq \beta .
\end{aligned}
$$

where,

$$
h_{m}(x)=\left(\begin{array}{ll}
-\frac{1}{m+1} x^{m+1} & , m \neq-1 \\
-\log x & , m=-1
\end{array}\right.
$$

and $\quad g_{m}(x)=h_{m}(x)-h_{m}(1), x \in[0,1)$.

If $m_{i}=0, \quad i=1,2, \ldots, n-1$ and $k=1$, then $X_{d}(r, n, m, k)$ reduces to the $(n-r+1)^{\text {th }}$ lower order statistic, i.e. $X_{n-r+1: n}$ from the sample $X_{1}, X_{2}, \ldots, X_{n}$ [David and Nagaraja (2003)]. If $m_{i}=m \rightarrow-1, i=1,2, \ldots, n-1$ and $k=1$, then $X_{d}(r, n, m, k)$ is the lower record value from an infinite sequence of independent and identically distributed (iid) random variables $(r v)$ [Ahsanullah (1995)].

Cardeiro et al.(2013) proposed a new class of distributions and discussed some of its properties. The $c d f$ of this newly proposed class of distribution is

$$
F(x)=\left[1-\{1-H(x)\}^{\alpha}\right]^{\beta},
$$

and corresponding $p d f$

$$
f(x)=\alpha \beta[1-H(x)]^{\alpha-1}\left[1-\{1-H(x)\}^{\alpha}\right]^{\beta-1} h(x)
$$

where $\alpha>0$ and $\beta>0$ are the shape parameters and $H(x)$ is the $c d f$ of base distribution.

In view of (6) and (7), it can be seen that

$$
\frac{F(x)}{f(x)}=\left\{\frac{1}{\alpha \beta \lambda(x)} \sum_{t=1}^{\infty}\left(\begin{array}{c}
{[\alpha+t-1]} \\
t
\end{array}\right)[H(x)]^{t}\right\},
$$

where $\lambda(x)=\frac{h(x)}{1-H(x)}$ is hazard rate function and [.] is an integer.

The organization of paper is as given below: 
In section 2, relations between signle moment of $d g o s$ are studied and particular cases and examples based on it are presented. Section 3, relates to the product moments of $d g o s$, its particular cases and examples based on it. The characterization of the distribution under consideration through moments properties are presented in Section 4. Some concluding remarks and futuer recommendations are given in Section 5. At the end bibliography is given.

\section{Single Moment}

Theorem 2.1. Let Case I be satisfied. For exponentiated generalized class of distributions with $c d f$ as given in (6) and $n \in \square, \tilde{m} \in \square, k>0,1 \leq r \leq n$ with $j=1,2, \ldots$

$$
E\left[X_{d}^{j}(r, n, \tilde{m}, k)\right]-E\left[X_{d}^{j}(r-1, n, \tilde{m}, k)\right]=-\frac{j}{\alpha \beta \gamma_{r}} \sum_{t=1}^{\infty}\left(\begin{array}{c}
{[\alpha+t-1]} \\
t
\end{array}\right) E\left[B^{t}\left\{X_{d}(r, n, \tilde{m}, k)\right\}\right],
$$

where $\quad B^{t}(x)=\frac{x^{j-1}(H(x))^{t}}{\lambda(x)}, t=1,2, \ldots$ and $\lambda(x)=\frac{h(x)}{1-H(x)}$.

Proof. For dgos Athar et al. (2008) have shown that

$$
E\left[\xi\left\{X_{d}(r, n, \tilde{m}, k)\right\}\right]-E\left[\xi\left\{X_{d}(r-1, n, \tilde{m}, k)\right\}\right]=-C_{r-2} \int_{-\infty}^{\infty} \xi^{\prime}(x) \sum_{i=1}^{r} a_{i}(r)[F(x)]^{\gamma_{i}} d x .
$$

Consider $\xi(x)=x^{j}$ in the above relation, then we have

$$
E\left[X_{d}^{j}(r, n, \tilde{m}, k)\right]-E\left[X_{d}^{j}(r-1, n, \tilde{m}, k)\right]=-j C_{r-2} \int_{-\infty}^{\infty} x^{j-1} \sum_{i=1}^{r} a_{i}(r)[F(x)]^{\gamma_{i}} d x .
$$

Now on application of relation (8) in (10), we get

$$
\begin{aligned}
& E\left[X_{d}^{j}(r, n, \tilde{m}, k)\right]-E\left[X_{d}^{j}(r-1, n, \tilde{m}, k)\right] \\
& \quad=-\frac{j C_{r-1}}{\gamma_{r}} \int_{-\infty}^{\infty} x^{j-1} \sum_{i=1}^{r} a_{i}(r)[F(x)]^{\gamma_{i}-1}\left\{\frac{1}{\alpha \beta \lambda(x)} \sum_{t=1}^{\infty}([\alpha+t-1])[H(x)]^{t} f(x)\right\} d x \\
& \quad=-\frac{j}{\alpha \beta \gamma_{r}} \sum_{t=1}^{\infty}\left(\begin{array}{c}
{[\alpha+t-1]} \\
t
\end{array}\right) C_{r-1} \int_{-\infty}^{\infty} B^{t}(x) \sum_{i=1}^{r} a_{i}(r)[F(x)]^{\gamma_{i}-1} f(x) d x,
\end{aligned}
$$

which leads to (9).

Corollary 2.1: Under the conditions as stated in Theorem 2.1 with $\alpha=1$, the relation between moment of dgos from Lehman type I is given as

$$
E\left[X_{d}^{j}(r, n, \tilde{m}, k)\right]-E\left[X_{d}^{j}(r-1, n, \tilde{m}, k)\right]=-\frac{j}{\beta \gamma_{r}} E\left[\psi\left\{X_{d}(r, n, \tilde{m}, k)\right\}\right],
$$

where $\quad \psi(x)=\frac{H(x)}{h(x)} x^{j-1}$.

Corollary 2.2: Under the conditions as stated in Theorem 2.1 with $\beta=1$, the relation between moment of $d g o s$ from Lehman type II is

$$
E\left[X_{d}^{j}(r, n, \tilde{m}, k)\right]-E\left[X_{d}^{j}(r-1, n, \tilde{m}, k)\right]=-\frac{j}{\alpha \gamma_{r}} \sum_{t=1}^{\infty}\left(\begin{array}{c}
{[\alpha+t-1]} \\
t
\end{array}\right) E\left[B^{t}\left\{X_{d}(r, n, \tilde{m}, k)\right\}\right],
$$

Theorem 2.2: Let Case II be satisfied. Under the conditions as stated in Theorem 2.1

$$
E\left[X_{d}^{j}(r, n, m, k)\right]-E\left[X_{d}^{j}(r-1, n, m, k)\right]=-\frac{j}{\alpha \beta \gamma_{r}} \sum_{t=1}^{\infty}\left(\begin{array}{c}
{[\alpha+t-1]} \\
t
\end{array}\right) E\left[B^{t}\{X(r, n, m, k)\}\right] .
$$

Proof. The relation (13) can be established in view of Athar at el. (2008) and (8) on the lines of Theorem 2.1. 
Remark 2.1: The recurrence relation for the single moments of reverse order statistics (at $m=0$ and $k=1$ ) is given by

$$
\left.E\left(X_{n-r+1: n}^{j}\right)=E\left(X_{n-r+2: n}^{j}\right)-\frac{j}{(n-r+1) \alpha \beta} \sum_{t=1}^{\infty}\left(\begin{array}{c}
{[\alpha+t-1]} \\
t
\end{array}\right) E\left[B^{t}\left(X_{n-r+1: n}\right)\right\}\right] .
$$

Further, the relation for single moment of order statistics can be obtained by replacing $(n-r+1)$ with $(r-1)$

$$
\left.E\left(X_{r: n}^{j}\right)-E\left(X_{r-1: n}^{j}\right)=-\frac{j}{(r-1) \alpha \beta} \sum_{t=1}^{\infty}\left(\begin{array}{c}
{[\alpha+t-1]} \\
t
\end{array}\right) E\left[B^{t}\left(X_{r-1: n}\right)\right\}\right] .
$$

Remark 2.2: The recurrence relation for the single moments of $k^{\text {th }}$ lower record value is

$$
E\left(X_{u(r)}^{(k)}\right)^{j}=E\left(X_{u(r-1)}^{(k)}\right)^{j}-\frac{j}{k \alpha \beta} \sum_{l=1}^{\infty}\left(\begin{array}{c}
{[\alpha+t-1]} \\
t
\end{array}\right) E\left[B^{t}\left(X_{u(r)}^{(k)}\right)\right] .
$$

\subsection{Examples}

\subsubsection{Exponentiated generalized Fréchet distribution}

Let the base distribution is Fréchet distribution with $c d f$

$$
H(x)=\exp \left[-(\sigma / x)^{v}\right], \quad x>0 ; \sigma, v>0,
$$

and the corresponding $p d f$

$$
h(x)=v \sigma^{v} x^{-v-1} \exp \left[-(\sigma / x)^{v}\right], x>0 ; \sigma, v>0
$$

Then the $c d f$ of the exponentiated generalized Fréchet distribution is

$$
F(x)=\left[1-\left\{1-\exp \left[-(\sigma / x)^{v}\right]\right\}^{\alpha}\right]^{\beta}, \quad \alpha, \beta, \sigma, v>0 .
$$

Now, we have

$$
\begin{aligned}
B^{t}(x) & =x^{j-1}\left(\frac{H^{t}(x)\{1-H(x)\}}{h(x)}\right) \\
& =\frac{x^{j+v}}{v \sigma^{v}} e^{-(t-1)(\sigma / x)^{v}}\left(1-e^{-(\sigma / x)^{v}}\right) \\
& =\frac{x^{j+v}}{v \sigma^{v}}\left(\sum_{d=0}^{\infty}(-1)^{d} \frac{\sigma^{v d} x^{-v d}(t-1)^{d}}{d !}\right)\left(\sum_{a=0}^{\infty}(-1)^{a} \frac{\sigma^{v(a+1)} x^{-v(a+1)}}{(a+1) !}\right) \\
& =\frac{1}{v} \sum_{a=0}^{\infty} \sum_{d=0}^{\infty}(-1)^{a+d} \frac{\sigma^{v(a+d)}(t-1)^{d}}{d !(a+1) !} x^{j+v(1-a-d)-1}
\end{aligned}
$$

Thus, in view of (9), we get

$$
\begin{aligned}
& E\left[X_{d}^{j}(r, n, \tilde{m}, k)\right]-E\left[X_{d}^{j}(r-1, n, \tilde{m}, k)\right]=-\frac{j}{v \alpha \beta \gamma_{r}} \sum_{t=1}^{\infty} \sum_{a=0}^{\infty} \sum_{d=0}^{\infty}(-1)^{a+d}\left(\begin{array}{c}
{[\alpha+t-1]} \\
t
\end{array}\right) \frac{\sigma^{v(a+d)}(t-1)^{d}}{d !(a+1) !} \\
& \times E\left[X_{d}^{j+v(1-a-d)-1}(r, n, \tilde{m}, k)\right]
\end{aligned}
$$

Further at $\alpha=1$, we have

$$
\psi(x)=\frac{H(x)}{h(x)} x^{j-1}=\frac{1}{v \sigma^{v}} x^{j+v} .
$$


Thus, from (11), we get the relation between moment of $d g o s$ from exponentiated Fréchet distribution as

$$
E\left[X_{d}^{j}(r, n, \tilde{m}, k)\right]-E\left[X_{d}^{j}(r-1, n, \tilde{m}, k)\right]=-\frac{j}{v \sigma^{v} \beta \gamma_{r}} E\left[X_{d}^{j+v}(r, n, \tilde{m}, k)\right]
$$

and at $\beta=1$ in (17), we get the relation between moment of $d g o s$ from Fréchet distribution

$$
E\left[X_{d}^{j}(r, n, \tilde{m}, k)\right]-E\left[X_{d}^{j}(r-1, n, \tilde{m}, k)\right]=-\frac{j}{v \sigma^{v} \gamma_{r}} E\left[X_{d}^{j+v}(r, n, \tilde{m}, k)\right] .
$$

\subsubsection{Exponentiated generalized power function distribution}

Suppose the base distribution is power function distribution having $c d f$

$$
H(x)=x^{p}, 0<x<1, p>0
$$

and the corresponding $p d f$

$$
h(x)=p x^{p-1}, 0<x<1, p>0 .
$$

Thus, the $c d f$ of exponentiated generalized power function distribution is

$$
F(x)=\left[1-\left(1-x^{p}\right)^{\alpha}\right]^{\beta}, 0<x<1 ; \alpha, \beta>0 .
$$

Now, we have

$$
\begin{aligned}
B^{t}(x) & =x^{j-1}\left(\frac{H^{t}(x)-H^{t+1}(x)}{h(x)}\right) \\
& =x^{j-1}\left(\frac{x^{p t}-x^{p(t+1)}}{p x^{p-1}}\right) \\
& =\frac{1}{p}\left(x^{j+p(t-1)}-x^{j+p t}\right)
\end{aligned}
$$

Therefore, from (9) we get

$$
\begin{aligned}
E\left[X_{d}^{j}(r, n, \tilde{m}, k)\right]-E\left[X_{d}^{j}(r-1, n, \tilde{m}, k)\right]= & -\frac{j}{\alpha \beta p \gamma_{r}} \sum_{t=1}^{\infty}\left(\begin{array}{c}
{[\alpha+t-1]} \\
t
\end{array}\right) \\
& \times\left[E\left[X_{d}^{j+p(t-1)}(r, n, \tilde{m}, k)\right]-E\left[X_{d}^{j+p t}(r, n, \tilde{m}, k)\right]\right]
\end{aligned}
$$

Since at $\alpha=1$, we have

$$
\psi(x)=\frac{H(x)}{h(x)} x^{j-1}=\frac{1}{p} x^{j} .
$$

Thus, using (11), we get the relation for moment of $d g o s$ from exponentiated power function distribution as

$$
E\left[X_{d}^{j}(r, n, \tilde{m}, k)\right]-E\left[X_{d}^{j}(r-1, n, \tilde{m}, k)\right]=-\frac{j}{p \beta \gamma_{r}} E\left[X_{d}^{j}(r, n, \tilde{m}, k)\right] .
$$

Further, at $\beta=1$, we get the relation for moment of $d g o s$ from power function distribution as

$$
E\left[X_{d}^{j}(r, n, \tilde{m}, k)\right]-E\left[X_{d}^{j}(r-1, n, \tilde{m}, k)\right]=-\frac{j}{p \gamma_{r}} E\left[X_{d}^{j}(r, n, \tilde{m}, k)\right] .
$$

The relation (21) is also obtained by Athar and Faizan (2011). 


\subsubsection{Exponentiated generalized Pareto distribution}

Let the parent distribution is Pareto distribution with $c d f$

$$
H(x)=1-v^{\theta} x^{-\theta}, v<x<\infty ; v>0, \theta>1 .
$$

and the corresponding $p d f$

$$
h(x)=\theta v^{\theta} x^{-(\theta+1)}, v<x<\infty ; v>0, \theta>1 .
$$

Thus, the $c d f$ of the exponentiated generalized Pareto distribution is given by

$$
F(x)=\left[1-v^{\alpha \theta} x^{-\alpha \theta}\right]^{\beta}, v<x<\infty ; v>0, \theta>1 .
$$

Now,

$$
\begin{aligned}
B^{t}(x) & =x^{j-1}\left\{\frac{H^{t}(x)[1-H(x)]}{h(x)}\right\}=\frac{1}{\theta} x^{j}\left(1-v^{\theta} x^{-\theta}\right)^{t} \\
& =\frac{1}{\theta} \sum_{l=0}^{t}(-1)^{l}\left(\begin{array}{l}
t \\
l
\end{array}\right) v^{\theta l} x^{j-\theta l}
\end{aligned}
$$

Therefore, using (9), we get

$$
E\left[X_{d}^{j}(r, n, \tilde{m}, k)\right]-E\left[X_{d}^{j}(r-1, n, \tilde{m}, k)\right]=-\frac{j}{\alpha \beta \theta \gamma_{r}} \sum_{t=1}^{\infty} \sum_{l=0}^{t}(-1)^{l}\left(\begin{array}{c}
{[\alpha+t-1]} \\
t
\end{array}\right)\left(\begin{array}{l}
t \\
l
\end{array}\right) v^{\theta l} E\left[X_{d}^{j-\theta l}(r, n, \tilde{m}, k)\right]
$$

Now, at $\alpha=1$, we have

$$
\psi(x)=\frac{H(x)}{h(x)} x^{j-1}=\frac{1}{\theta v^{\theta}} x^{j+\theta}-\frac{1}{\theta} x^{j} .
$$

Thus, in view of (11), we get the relation for moment of dgos from exponentiated Pareto distribution as

$$
\left(\beta \theta \gamma_{r}-j\right) E\left[X_{d}^{j}(r, n, \tilde{m}, k)\right]=\beta \theta \gamma_{r} E\left[X_{d}^{j}(r-1, n, \tilde{m}, k)\right]-\frac{j}{v^{\theta}} E\left[X_{d}^{j+\theta}(r, n, \tilde{m}, k)\right]
$$

The recursive relation for moment of $d g o s$ from exponentiated Pareto distribution with different form of $p d f$ is also obtained by Khan and Kumar (2010).

Further at $\beta=1$ in (23), we get the relation for moment of $d g o s$ from Pareto distribution.

\subsubsection{Exponentiated generalized Weibull distribution}

Let the parent distribution is Weibull distribution distribution having $c d f$

$$
H(x)=1-e^{-(x / \theta)^{\lambda}}, x>0 ; \theta, \lambda>0,
$$

and the corresponding $p d f$ is

$$
h(x)=\frac{\lambda}{\theta}\left(\frac{x}{\theta}\right)^{\lambda-1} e^{-(x / \theta)^{\lambda}}, x>0 ; \theta, \lambda>0 .
$$

Therefore, the $c d f$ of exponentiated generalized Weibull distribution is

$$
F(x)=\left[1-e^{-\alpha(x / \theta)^{\lambda}}\right]^{\beta}, x>0 ; \alpha, \beta>0 .
$$

Now, we have 


$$
\begin{aligned}
B^{t}(x) & =x^{j-1}\left\{\frac{\theta^{\lambda}}{\lambda} x^{-\lambda+1}\left(1-e^{-(x / \theta)^{\lambda}}\right)^{t}\right\}=\frac{\theta^{\lambda}}{\lambda} x^{j-\lambda}\left(1-e^{-(x / \theta)^{\lambda}}\right)^{t} \\
& =\frac{\theta^{\lambda}}{\lambda} x^{j-\lambda} \sum_{v=0}^{t}(-1)^{\nu}\left(\begin{array}{c}
t \\
v
\end{array}\right) e^{-v(x / \theta)^{\lambda}} \\
& =\frac{\theta^{\lambda}}{\lambda} x^{j-\lambda} \sum_{v=0}^{t} \sum_{a=0}^{\infty} \frac{(-1)^{v+a} v^{a}}{a ! \theta^{a \lambda}}\left(\begin{array}{c}
t \\
v
\end{array}\right) x^{a \lambda} \\
& =\frac{1}{\lambda} \sum_{v=0}^{t} \sum_{a=0}^{\infty} \frac{(-1)^{v+a}}{a !} v^{a} \theta^{-\lambda(a-1)}\left(\begin{array}{c}
t \\
v
\end{array}\right) x^{j+\lambda(a-1)}
\end{aligned}
$$

Then from (9) we get

$$
\begin{aligned}
E\left[X_{d}^{j}(r, n, \tilde{m}, k)\right]-E\left[X_{d}^{j}(r-1, n, \tilde{m}, k)\right]= & -\frac{j}{\lambda \alpha \beta \gamma_{r}} \sum_{t=1}^{\infty} \sum_{v=0}^{t} \sum_{a=0}^{\infty} \frac{(-1)^{v+a}}{a !} v^{a} \theta^{-\lambda(a-1)} \\
& \times\left(\begin{array}{c}
{[\alpha+t-1]} \\
t
\end{array}\right)\left(\begin{array}{c}
t \\
v
\end{array}\right) E\left[X_{d}^{j+\lambda(a-1)}(r, n, \tilde{m}, k)\right] .
\end{aligned}
$$

Now, at $\alpha=1$, we have

$$
\psi(x)=\frac{H(x)}{h(x)} x^{j-1}=\frac{1}{\lambda} \sum_{i=1}^{\infty} \frac{1}{i} \frac{x^{j+\lambda(i-1)}}{\theta^{\lambda(i-1)}} .
$$

Thus, in view of (11), we get the relation for moment of $d g o s$ from exponentiated Weibull distribution as

$$
E\left[X_{d}^{j}(r, n, \tilde{m}, k)\right]-E\left[X_{d}^{j}(r-1, n, \tilde{m}, k)\right]=-\frac{j}{\beta \lambda \gamma_{r}} \sum_{i=1}^{\infty} \frac{1}{i ! \theta^{\lambda(i-1)}} E\left[X_{d}^{j+\lambda(i-1)}(r, n, \tilde{m}, k)\right] .
$$

Similar result is also obtained by Khan et al. (2008).

Further, at $\beta=1$ in (25), we get the relation for moment of dgos for Weibull distribution.

Similarly, several recurrence relations for single moment of dgos from exponentiated generalized distributions for the given parent distributions can be established using Theorem 2.1.

\section{Product Moment}

Theorem 3.1: Suppose Case I be satisfied. For exponentiated generalized class of distributions as given in (6). Fix a positive integer $k$ and for $n \in N, \tilde{m} \in R, 1 \leq r<s \leq n, i, j=1,2, \ldots$

$$
\begin{aligned}
E\left[X_{d}^{i}(r, n, \tilde{m}, k) X_{d}^{j}(s, n, \tilde{m}, k)\right]-E\left[X_{d}^{i}(r, n, \tilde{m}, k) X_{d}^{j}(s-1, n, \tilde{m}, k)\right] & \\
& =-\frac{j}{\gamma_{s} \alpha \beta} \sum_{t=1}^{\infty}\left(\begin{array}{c}
{[\alpha+t-1]} \\
t
\end{array}\right) E\left[B^{t}\left\{X_{d}(r, n, \tilde{m}, k) \cdot X_{d}(s, n, \tilde{m}, k)\right\}\right],
\end{aligned}
$$

where, $B^{t}(x, y)=\frac{x^{i} y^{j-1}(H(y))^{t}}{\lambda(y)}$ and $\lambda(y)=\frac{h(y)}{1-H(y)}$.

Proof. We have, by Athar et al. (2008),

$$
\begin{aligned}
& E\left[\xi\left\{X_{d}(r, n, \tilde{m}, k), X_{d}(s, n, \tilde{m}, k)\right\}\right]- E\left[\xi\left\{X_{d}(r, n, \tilde{m}, k), X_{d}(s-1, n, \tilde{m}, k)\right\}\right] \\
&=-C_{s-2} \iint_{-\infty \leq y<x \leq \infty} \frac{\partial}{\partial y} \xi(x, y)\left(\sum_{i=r+1}^{s} a_{i}^{(r)}(s)\left[\frac{F(y)}{F(x)}\right]^{\gamma_{i}}\right) \\
& \times\left(\sum_{i=1}^{r} a_{i}(r)[F(x)]^{\gamma_{i}}\right) \frac{f(x)}{F(x)} d y d x .
\end{aligned}
$$


Now consider $\xi(x, y)=\xi_{1}(x) \cdot \xi_{2}(y)=x^{i} \cdot y^{j}$ in (27), then we have

$$
\begin{aligned}
& E\left[X_{d}^{i}(r, n, \tilde{m}, k) \cdot X_{d}^{j}(s, n, \tilde{m}, k)\right]-E\left[X_{d}^{i}(r, n, \tilde{m}, k) . X_{d}^{j}(s-1, n, \tilde{m}, k)\right] \\
&=-j C_{s-2} \iint_{-\infty \leq y<x \leq \infty} x^{i} y^{j-1}\left(\sum_{i=r+1}^{s} a_{i}^{(r)}(s)\left[\frac{F(y)}{F(x)}\right]^{\gamma_{i}}\right) \\
& \times\left(\sum_{i=1}^{r} a_{i}(r)[F(x)]^{\gamma_{i}}\right) \frac{f(x)}{F(x)} d y d x .
\end{aligned}
$$

Now using (8) in (28), we get

$$
\begin{aligned}
E\left[X_{d}^{i}(r, n, \tilde{m}, k) . X_{d}^{j}\right. & (s, n, \tilde{m}, k)]-E\left[X_{d}^{i}(r, n, \tilde{m}, k) \cdot X_{d}^{j}(s-1, n, \tilde{m}, k)\right] \\
= & -\frac{j C_{s-1}}{\gamma_{s} \alpha \beta} \iint_{-\infty \leq y<x \leq \infty} x^{i} y^{j-1} \sum_{i=r+1}^{s} a_{i}^{(r)}(s)\left[\frac{F(y)}{F(x)}\right]^{\gamma_{i}} \\
& \times \sum_{i=1}^{r} a_{i}(r)[F(x)]^{\gamma_{i}} \frac{f(x)}{F(x)} \frac{f(y)}{F(y)}\left\{\frac{1}{\lambda(y)} \sum_{t=1}^{\infty}\left(\begin{array}{c}
{[\alpha+t-1]} \\
t
\end{array}\right)[H(y)]^{t}\right\} d y d x,
\end{aligned}
$$

which leads to (26).

Corollary 3.1: Under the conditions as stated in Theorem 3.1 with $\alpha=1$, the relation between product moment of dgos from Lehman type I distribution is given as

$$
\begin{aligned}
E\left[X_{d}^{i}(r, n, \tilde{m}, k) X_{d}^{j}(s, n, \tilde{m}, k)\right]-E\left[X_{d}^{i}(r, n, \tilde{m}, k) X_{d}^{j}(s-1, n, \tilde{m}, k)\right] & \\
& =-\frac{j}{\gamma_{s} \beta} E\left[\psi\left\{X_{d}(r, n, \tilde{m}, k) . X_{d}(s, n, \tilde{m}, k)\right\}\right],
\end{aligned}
$$

where $\psi(x, y)=\frac{H(y)}{h(y)} x^{i} y^{j-1}$.

Corollary 3.2: Under the conditions as stated in Theorem 3.1 with $\beta=1$, the relation between product moment of dgos from Lehman type II distribution is given as

$$
\begin{aligned}
E\left[X_{d}^{i}(r, n, \tilde{m}, k) X_{d}^{j}(s, n, \tilde{m}, k)\right]-E\left[X_{d}^{i}(r, n, \tilde{m}, k) X_{d}^{j}(s-1, n, \tilde{m}, k)\right] \\
\quad=-\frac{j}{\gamma_{s} \alpha} \sum_{t=1}^{\infty}\left(\begin{array}{c}
{[\alpha+t-1]} \\
t
\end{array}\right) E\left[B^{t}\left\{X_{d}(r, n, \tilde{m}, k) \cdot X_{d}(s, n, \tilde{m}, k)\right\}\right],
\end{aligned}
$$

where $B^{t}(x, y)$ is defined as earlier.

Theorem 3.2: Let Case II be satisfied. Then under the conditions as stated in Theorem 3.1

$$
\begin{aligned}
& E\left[X_{d}^{i}(r, n, m, k) X_{d}^{j}(s, n, m, k)\right]-E\left[X_{d}^{i}(r, n, m, k) X_{d}^{j}(s-1, n, m, k)\right] \\
& =-\frac{j}{\gamma_{s} \alpha \beta} \sum_{t=1}^{\infty}\left(\begin{array}{c}
{[\alpha+t-1]} \\
t
\end{array}\right) E\left[B^{t}\left\{X_{d}(r, n, m, k) . X_{d}(s, n, m, k)\right\}\right],
\end{aligned}
$$

where $B^{t}(x, y)$ is defined as earlier.

Proof. The relation (31) can be established in view of Athar at el. (2008) and (8) on the lines of Theorem 3.1. 
Remark 3.1: The relation for product moment of reverse order statistics can be obtained by putting $m=0, k=1$ in equation (31), whereas the relation for product moment of lower record values can be seen by letting $m \rightarrow-1$ in (31).

\subsection{EXAMPLES}

\subsubsection{Exponentiated generalized Fréchet distribution}

For the $c d f$ of exponentiated generalized Fréchet distribution as given in (16), we have

$$
B^{t}(x, y)=\frac{1}{v} \sum_{a=0}^{\infty} \sum_{d=0}^{\infty}(-1)^{a+d} \frac{\sigma^{\nu(a+d)}(t-1)^{d}}{d !(a+1) !} x^{i} y^{j+v(1-a-d)-1}
$$

Thus, in view of (26), we get

$$
\begin{aligned}
& E\left[X_{d}^{i}(r, n, \tilde{m}, k) X_{d}^{j}(s, n, \tilde{m}, k)\right]-E\left[X_{d}^{i}(r, n, \tilde{m}, k) X_{d}^{j}(s-1, n, \tilde{m}, k)\right] \\
& \quad=-\frac{j}{\gamma_{s} \alpha \beta v} \sum_{t=1}^{\infty} \sum_{a=0}^{\infty} \sum_{d=0}^{\infty}(-1)^{a+d}\left(\begin{array}{c}
{[\alpha+t-1]} \\
t
\end{array}\right) \frac{\sigma^{\nu(a+d)}(t-1)^{d}}{d !(a+1) !} E\left[X_{d}^{i}(r, n, \tilde{m}, k) X_{d}^{j+v(1-a-d)-1}(s, n, \tilde{m}, k)\right]
\end{aligned}
$$

At $\alpha=1$, we have

$$
\psi(x, y)=\frac{H(y)}{h(y)} x^{i} y^{j-1}=\frac{1}{v \sigma^{v}} x^{i} y^{j+v} .
$$

Therefore, in view of (29), the relation between product moment of dgos from exponentiated Fréchet distribution is given as

$$
\begin{array}{r}
E\left[X_{d}^{i}(r, n, \tilde{m}, k) X_{d}^{j}(s, n, \tilde{m}, k)\right]-E\left[X_{d}^{i}(r, n, \tilde{m}, k) X_{d}^{j}(s-1, n, \tilde{m}, k)\right] \\
=-\frac{j}{v \sigma^{v} \beta \gamma_{s}} E\left[X_{d}^{i}(r, n, \tilde{m}, k) X_{d}^{j+v}(s, n, \tilde{m}, k)\right] .
\end{array}
$$

Further, at $\beta=1$ in (32), we get the relation between product moment of $d g o s$ from Fréchet distribution.

\subsubsection{Exponentiated generalized power function distribution}

Let the $c d f$ of exponentiated generalized power function distribution is as given in (19). Then, we have

$$
B^{t}(x, y)=\frac{x^{i}}{p}\left(y^{j+p(t-1)}-y^{j+p t}\right)
$$

Thus, in view of (26), we have

$$
\begin{aligned}
E\left[X_{d}^{i}(r, n, \tilde{m}, k) X_{d}^{j}(s, n, \tilde{m}, k)\right]-E\left[X_{d}^{i}(r, n, \tilde{m}, k) X_{d}^{j}(s-1, n, \tilde{m}, k)\right] \\
\quad=\frac{j}{\alpha \beta p \gamma_{s}} \sum_{t=1}^{\infty}\left(\begin{array}{c}
{[\alpha+t-1]} \\
t
\end{array}\right)\left\{E\left[X_{d}^{i}(r, n, \tilde{m}, k) X_{d}^{j+p t}(s, n, \tilde{m}, k)\right]-E\left[X_{d}^{i}(r, n, \tilde{m}, k) X_{d}^{j+p(t-1)}(s, n, \tilde{m}, k)\right]\right\}
\end{aligned}
$$

At $\alpha=1$, we have

$$
\psi(x, y)=\frac{H(y)}{h(y)} x^{i} y^{j-1}=\frac{1}{p} x^{i} y^{j} .
$$

Thus, in view of (29), we get the relation for product moment of dgos from exponentiated power function distribution whereas with $\alpha=\beta=1$, we get the relation for power function distribution. 


\subsubsection{Exponentiated generalized Pareto distribution}

For exponentiated generalized Pareto distribution as given in (22), we have

$$
\begin{gathered}
B^{t}(x, y)=x^{i} y^{j-1}\left\{\frac{H^{t}(y)[1-H(y)]}{h(y)}\right\}=\frac{1}{\theta} \sum_{l=0}^{t}(-1)^{l}\left(\begin{array}{l}
t \\
l
\end{array}\right) v^{\theta l} x^{i} y^{j-\theta l} \\
E\left[X_{d}^{j}(r, n, \tilde{m}, k)\right]-E\left[X_{d}^{j}(r-1, n, \tilde{m}, k)\right]=-\frac{j}{\alpha \beta \theta \gamma_{r}} \sum_{t=1}^{\infty} \sum_{l=0}^{t}(-1)^{l}\left(\begin{array}{c}
{[\alpha+t-1]} \\
t
\end{array}\right)\left(\begin{array}{l}
t \\
l
\end{array}\right) v^{\theta l} E\left[X_{d}^{j-\theta l}(r, n, \tilde{m}, k)\right]
\end{gathered}
$$

Therefore, in view of (26), we get

$$
\begin{aligned}
E\left[X_{d}^{i}(r, n, \tilde{m}, k) X_{d}^{j}(s, n, \tilde{m}, k)\right]-E\left[X_{d}^{i}(r, n, \tilde{m}, k) X_{d}^{j}(s-1, n, \tilde{m}, k)\right] & \\
= & -\frac{j}{\alpha \beta \theta \gamma_{s}} \sum_{t=1}^{\infty} \sum_{l=0}^{t}(-1)^{l}\left(\begin{array}{c}
\alpha+t-1] \\
t
\end{array}\right)\left(\begin{array}{c}
t \\
l
\end{array}\right) v^{\theta l} E\left[X_{d}^{i}(r, n, \tilde{m}, k) X_{d}^{j-\theta l}(s, n, \tilde{m}, k)\right] .
\end{aligned}
$$

Further, at $\alpha=1$ and in view of (29), we get the relation for product moment of dgos for exponentiated Pareto distribution. Similar result is also obtained by Khan and Kumar (2010) with different form of $p d f$, whereas at $\alpha=\beta=1$, we have the relation for product moment of $d g o s$ from Pareto distribution.

\subsubsection{Exponentiated generalized Weibull distribution}

Let the $c d f$ of exponentiated generalized Weibull distribution has the form as given in (24).

Therefore, we have

$$
B^{t}(x, y)=\frac{1}{\lambda} \sum_{v=0}^{t} \sum_{a=0}^{\infty} \frac{(-1)^{v+a}}{a !} v^{a} \theta^{-\lambda(a-1)}\left(\begin{array}{l}
t \\
v
\end{array}\right) x^{i} y^{j+\lambda(a-1)}
$$

Now using relation (26), we get

$$
\begin{aligned}
& E\left[X_{d}^{i}(r, n, \tilde{m}, k) X_{d}^{j}(s, n, \tilde{m}, k)\right]-E\left[X_{d}^{i}(r, n, \tilde{m}, k) X_{d}^{j}(s-1, n, \tilde{m}, k)\right] \\
& \quad=-\frac{j}{\lambda \alpha \beta \gamma_{s}} \sum_{t=1}^{\infty} \sum_{v=0}^{t} \sum_{a=0}^{\infty} \frac{(-1)^{v+a}}{a !} v^{a} \theta^{-\lambda(a-1)}\left(\begin{array}{c}
\alpha+t-1] \\
t
\end{array}\right)\left(\begin{array}{c}
t \\
v
\end{array}\right) E\left[X_{d}^{i}(r, n, \tilde{m}, k) X_{d}^{j+\lambda(a-1)}(s, n, \tilde{m}, k)\right]
\end{aligned}
$$

Now, at $\alpha=1$, we have

$$
\psi(x, y)=\frac{H(x)}{h(x)} x^{i} y^{j-1}=\frac{1}{\lambda} \sum_{p=1}^{\infty} \frac{1}{p !} \frac{x^{i} y^{j+\lambda(p-1)}}{\theta^{\lambda(p-1)}} .
$$

Thus, in view of (29), we get the relation for product moment of $d g o s$ from exponentiated Weibull distribution as given by Khan et al. (2008) and when $\alpha=\beta=1$, we get the relation for product moments of $d g o s$ from Weibull distribution.

Similarly, several recurrence relations for product moment of dgos from exponentiated generalized distributions for given parent distributions can be established using Theorem 3.1.

\section{CHARACTERIZATION}

Theorem 4.1: Let $X$ be a continuous random variable having an absolutely continuous distribution function $F(x)$, $0<F(x)<1$ for all $-\infty<x<\infty$ and $E\left|X^{j}\right|<\infty$ for some $j \geq 1$. Further assume that $n_{i}, i=1,2, \ldots, n$ be a sequence of integers satisfying $2 \leq n_{1}<n_{2}<\ldots$ and $\sum_{i=1}^{\infty} \frac{1}{n_{i}}=\infty$. Then for $1 \leq r \leq n$ and $k \geq 1$ 


$$
E\left[X_{d}^{j}(r, n, \tilde{m}, k)\right]-E\left[X_{d}^{j}(r-1, n, \tilde{m}, k)\right]=-\frac{j}{\alpha \beta \gamma_{r}} \sum_{t=1}^{\infty}\left(\begin{array}{c}
{[\alpha+t-1]} \\
t
\end{array}\right) E\left[B^{t}\left\{X_{d}(r, n, \tilde{m}, k)\right\}\right]
$$

where $\quad B^{t}(x)=\frac{x^{j-1}(H(x))^{t}}{\lambda(x)}$ and $\lambda(x)=\frac{h(x)}{1-H(x)}$.

if and only if

$$
F(x)=\left[1-\{1-H(x)\}^{\alpha}\right]^{\beta},-\infty<x<\infty ; \alpha, \beta>0 .
$$

Proof. The necessary part follows immediately from (9). On the other hand, suppose the relation in (33) is satisfied, then on using Athar et al. (2008) for $\xi(x)=x^{j}$, we have

$$
\begin{aligned}
-j C_{r-2} \int_{-\infty}^{\infty} x^{j-1} \sum_{i=1}^{r} a_{i}(r)[F(x)]^{\gamma_{i}} d x= & -\frac{j}{\alpha \beta \gamma_{r}} \sum_{t=1}^{\infty}\left(\begin{array}{c}
{[\alpha+t-1]} \\
t
\end{array}\right) C_{r-1} \int_{-\infty}^{\infty} B^{t}(x) \sum_{i=1}^{r} a_{i}(r)[F(x)]^{\gamma_{i}-1} f(x) d x \\
& =-\frac{j}{\alpha \beta \gamma_{r}} \sum_{t=1}^{\infty}\left(\begin{array}{c}
{[\alpha+t-1]} \\
t
\end{array}\right) C_{r-1} \int_{-\infty}^{\infty} \frac{x^{j-1}(H(x))^{t}}{\lambda(x)} \sum_{i=1}^{r} a_{i}(r)[F(x)]^{\gamma_{i}-1} f(x) d x,
\end{aligned}
$$

which implies,

$$
\frac{j C_{r-1}}{\gamma_{r}} \int_{-\infty}^{\infty} x^{j-1} \sum_{i=1}^{r} a_{i}(r)[F(x)]^{\gamma_{i}-1} f(x)\left\{\frac{F(x)}{f(x)}-\sum_{t=1}^{\infty}\left(\begin{array}{c}
{[\alpha+t-1]} \\
t
\end{array}\right) \frac{(H(x))^{t}}{\alpha \beta \lambda(x)}\right\} d x=0 .
$$

Applying the generalization of Müntz-Sza'sz theorem [see, for example, Hwang and Lin (1984)] to (35), we get

$$
\frac{F(x)}{f(x)}=\frac{1}{\alpha \beta \lambda(x)} \sum_{t=1}^{\infty}\left(\begin{array}{c}
{[\alpha+t-1]} \\
t
\end{array}\right)[H(x)]^{t}=\frac{1}{\alpha \beta} \frac{\left[1-\{1-H(x)\}^{\alpha}\right]}{\{1-H(x)\}^{\alpha-1} h(x)} .
$$

This implies that

$$
F(x)=\left[1-\{1-H(x)\}^{\alpha}\right]^{\beta},-\infty<x<\infty ; \alpha, \beta>0 .
$$

Hence Theorem 4.1 holds.

Theorem 4.2: Under the condsitions as stated in Thorem 4.1. Fix a positive integer $k$ and for $n \in N, \tilde{m} \in R$, $1 \leq r<s \leq n, i, j=1,2, \ldots$

$$
\begin{aligned}
E\left[X_{d}^{i}(r, n, \tilde{m}, k) X_{d}^{j}(s, n, \tilde{m}, k)\right]-E\left[X_{d}^{i}(r, n, \tilde{m}, k) X_{d}^{j}(s-1, n, \tilde{m}, k)\right] & \\
& =-\frac{j}{\gamma_{s} \alpha \beta} \sum_{t=1}^{\infty}\left(\begin{array}{c}
{[\alpha+t-1]} \\
t
\end{array}\right) E\left[B^{t}\left\{X_{d}(r, n, \tilde{m}, k) \cdot X_{d}(s, n, \tilde{m}, k)\right\}\right],
\end{aligned}
$$

where, $B^{t}(x, y)=\frac{x^{i} y^{j-1}(H(y))^{t}}{\lambda(y)}$ and $\lambda(y)=\frac{h(y)}{1-H(y)}$.

if and only if

$$
F(x)=\left[1-\{1-H(x)\}^{\alpha}\right]^{\beta},-\infty<x<\infty ; \alpha, \beta>0 .
$$

Proof. Necessary part follows from (26). On the other hand, if the relation (36) is satisfied, then in view of Athar $e t$ al. (2008) with $\xi(x, y)=x^{i} y^{j}$, we have 


$$
\begin{aligned}
& -j C_{s-2} \iint_{-\infty \leq y<x \leq \infty} x^{i} y^{j-1}\left(\sum_{i=r+1}^{s} a_{i}^{(r)}(s)\left[\frac{F(y)}{F(x)}\right]^{\gamma_{i}}\right)\left(\sum_{i=1}^{r} a_{i}(r)[F(x)]^{\gamma_{i}}\right) \frac{f(x)}{F(x)} d y d x \\
& =-\frac{j C_{s-1}}{\gamma_{s} \alpha \beta} \sum_{t=1}^{\infty}\left(\begin{array}{c}
{[\alpha+t-1]} \\
t
\end{array}\right) \int_{-\infty<y<x<\infty} B^{t}(x, y)\left(\sum_{i=r+1}^{s} a_{i}^{(r)}(s)\left[\frac{F(y)}{F(x)}\right]^{\gamma_{i}}\right) \\
& \times\left(\sum_{i=1}^{r} a_{i}(r)[F(x)]^{\gamma_{i}}\right) \frac{f(x)}{F(x)} \frac{f(y)}{F(y)} d y d x, \\
& \text { or } \quad \frac{j C_{s-1}}{\gamma_{s}} \int_{-\infty \leq y<x \leq \infty} x^{i} y^{j-1}\left(\sum_{i=r+1}^{s} a_{i}^{(r)}(s)\left[\frac{F(y)}{F(x)}\right]^{\gamma_{i}}\right)\left(\sum_{i=1}^{r} a_{i}(r)[F(x)]^{\gamma_{i}}\right) \frac{f(x)}{F(x)} d y d x \\
& =\frac{j C_{s-1}}{\gamma_{s} \alpha \beta} \sum_{t=1}^{\infty}\left(\begin{array}{c}
{[\alpha+t-1]} \\
t
\end{array}\right) \int_{-\infty<y<x<\infty} \frac{x^{i} y^{j-1}(H(y))^{t}}{\lambda(y)}\left(\sum_{i=r+1}^{s} a_{i}^{(r)}(s)\left[\frac{F(y)}{F(x)}\right]^{\gamma_{i}}\right) \\
& \times\left(\sum_{i=1}^{r} a_{i}(r)[F(x)]^{\gamma_{i}}\right) \frac{f(x)}{F(x)} \frac{f(y)}{F(y)} d y d x .
\end{aligned}
$$

This implies

$$
\begin{gathered}
\frac{j C_{s-1}}{\gamma_{s}} \int_{-\infty \leq y<x \leq \infty} x^{i} y^{j-1}\left(\sum_{i=r+1}^{s} a_{i}^{(r)}(s)\left[\frac{F(y)}{F(x)}\right]^{\gamma_{i}}\right)\left(\sum_{i=1}^{r} a_{i}(r)[F(x)]^{\gamma_{i}}\right) \frac{f(x)}{F(x)} \frac{f(y)}{F(y)} \\
\times\left\{\frac{F(y)}{f(y)}-\sum_{t=1}^{\infty}\left(\begin{array}{c}
{[\alpha+t-1]} \\
t
\end{array}\right) \frac{(H(y))^{t}}{\alpha \beta \lambda(y)}\right\} d y d x=0 .
\end{gathered}
$$

Applying the generalization of Müntz-Sza'sz theorem [see, for example, Hwang and Lin (1984)] to (38), we get

$$
\frac{F(y)}{f(y)}=\sum_{t=1}^{\infty}\left(\begin{array}{c}
{[\alpha+t-1]} \\
t
\end{array}\right) \frac{(H(y))^{t}}{\alpha \beta \lambda(y)},
$$

which leads to (37).

\section{Conclusions}

Burkschat et al. (2003) proposed a model that enables a common approach to descending ordered random variables like reverse order statistics, lower record values etc. and named it $d g o s$. Several authors studied moment properties of $d g o s$ for some specific distributions. Main aim of this paper to present a unified approach to study the moment properties of exponentiated distributions and their generalized forms by considering exponentiated generalized class of distributions as given by Cardeiro et al.(2013). This exponentiated generalized family gives greater flexibility of its tails and can be applied in many areas of engineering and biology. It is also evident that recurrence relations between moments reduce the amount of direct computation and can be used in characterization problems. This paper may be useful for those who work in the field of ordered random variable and distribution theory.

\section{References}

1. Ahsanullah, M. (1995). Record Statistics. Nova Science Publishers, New York.

2. Ahsanullah, M. (2004). A characterization of the uniform distribution by dual generalized order statistics. Comm. Statist. Theory Methods, 33, 2921-2928. 
3. Ahsanullah, M. (2005). On lower generalized order statistics and a characterization of power function distribution. Statistical Methods, 7(1), 16-28.

4. Athar, H., Anwar, Z. and Khan, R.U. (2008). On recurrence relations for the expectations of function of lower generalized order statistics. Pakistan. J. Statist., 24(2), 111-122.

5. Athar, H. and Faizan, M. (2011). Moments of lower generalized order statistics from power function distribution and its characterization. International Journal of Statistical Sciences, 11 (Special Issue), 125134.

6. Athar, H., Khan, R.U. and Anwar, Z. (2010). exact moments of lower generalized order statistics from power function distribution. Calcutta Stat. Assoc. Bull., 62 (1-2), 31-46.

7. Burkschat, M., Cramer, E. and Kamps, U. (2003). Dual generalized order statistics. Metron, LXI(1), 1326.

8. Cordeiro G.M., Ortega E.M.M and Cunha D.C.C. (2013). The exponentiated generalized class of distributions. J. Data Sci., 11(1), 1-27.

9. Daghistani, A.M., Al-Zahrani, B. and Shahbaz, M.Q. (2019). Relations for moments of dual generalized order statistics for a new inverse Kumaraswamy distribution. Pak. J. Stat. Oper. Res., 15(4), 989-997.

10. David, H.A. and Nagaraja, H.N. (2003). Order Statistics. John Wiley \& Sons, New York.

11. Hwang JS, Lin GD (1984). Extensions of Müntz-Szász theorems and application. Analysis. 4, 143-160.

12. Kamps, U. (1995). A Concept of Generalized Order Statistics. B.G. Teubner Stuttgart, Germany.

13. Khan, A.H., Anwar, Z. and Chishti, S. (2010). Characterization of continuous distributions through conditional expectation of functions of dual generalized order statistics. Pakistan J. Statist., 26(4), 615628.

14. Khan, M.A.R., Khan, R.U. and Singh, B. (2019). Relations for moments of dual generalized order statistics from exponentiated Rayleigh distribution and associated inference. J. Stat. Theory Appl, 18(4), 402-415.

15. Khan, R.U. and Khan, M.A. (2015). Dual generalized order statistics from family of J-shaped distribution and its characterization. Journal of King Saud University - Science, 27(4), 285-291.

16. Khan, R.U. and Kumar, D. (2010). On moments of lower generalized order statistics from exponentiated Pareto distribution and its characterization. Appl. Math. Sci., 4(55), 2711-2722.

17. Khan, R.U., Anwar, Z. and Athar, H. (2008). Recurrence relations for single and product moments of dual generalized order statistics from exponentiated Weibull distribution. Aligarh J. Statist., 28, 37-45.

18. Khwaja, S.K., Athar, H. and Nayabuddin (2012). Lower generalized order statistics from extended type-I generalized logistic distribution. Journal of Appl. Stat. Sc., 20(1), 21-28.

19. Kumar D. (2013). On moments of lower generalized order statistics from exponentiated Lomax distribution. American J. of Mathematical and Management Sciences, 32(4), 238-256.

20. Mbah, A.K. and Ahsanullah, M. (2007). Some characterization of the power function distribution based on lower generalized order statistics. Pakistan J. Statist., 23(2), 139-146.

21. Noor, Z., Akhter, Z. and Athar, H. (2015). On characterization of probability distributions through conditional expectation of generalized and dual generalized order statistics. Pakistan J. Statist., 31(2), $159-170$.

22. Pawlas, P. and Szynal, D. (2001). Recurrence relations for single and product moments of lower generalized order statistics from the inverse Weibull distribution. Demonstratio Mathematica, XXXIV(2), 353-358.

23. Shahbaz, M.Q., Ahsanullah, M., Shahbaz, S.H. and Al-Zahrani, B.M. (2016). Ordered Random Variables: Theory and Applications. Atlantis Press, Paris, France.

24. Singh, B., Khan, R.U. and Khan, A.N. (2021). Moments of dual generalized order statistics from Topp Leone weighted Weibull distribution and characterization. Ann. Data. Sci. (Online First). https://doi.org/10.1007/s40745-021-00324-1 